\title{
Association between tachyarrhythmia and mortality in a cohort of critically ill patients with coronavirus disease 2019 (COVID-19)
}

\author{
Peng Gao", Wei Wu ${ }^{1 \#}$, Ran Tian ${ }^{1}$, Xiaowei Yan $^{1}$, Hao Qian ${ }^{1}$, Fan Guo ${ }^{1}$, Taisheng Li $^{2}$, Zhengyin Liu ${ }^{2}$, \\ Jinglan Wang ${ }^{3}$, Xiang Zhou ${ }^{4}$, Yan Qin ${ }^{5}$, Dachun Zhao ${ }^{6}$, Xiuwu Bian ${ }^{7}$, Xue Lin ${ }^{1}$, Shuyang Zhang ${ }^{1}$ \\ ${ }^{1}$ Department of Cardiology, Peking Union Medical College Hospital, Chinese Academy of Medical Sciences and Peking Union Medical College, \\ Beijing, China; ${ }^{2}$ Department of Infectious Diseases, Peking Union Medical College Hospital, Chinese Academy of Medical Sciences and Peking Union \\ Medical College, Beijing, China; ${ }^{3}$ Department of Pulmonary and Critical Care Medicine, Peking Union Medical College Hospital, Chinese Academy \\ of Medical Sciences and Peking Union Medical College, Beijing, China; ${ }^{4}$ Department of Intensive Care Medicine, Peking Union Medical College \\ Hospital, Chinese Academy of Medical Sciences and Peking Union Medical College, Beijing, China; ${ }^{5}$ Department of Nephrology, Peking Union \\ Medical College Hospital, Chinese Academy of Medical Sciences and Peking Union Medical College, Beijing, China; ${ }^{6}$ Department of Pathology, \\ Peking Union Medical College Hospital, Chinese Academy of Medical Sciences and Peking Union Medical College, Beijing, China; ${ }^{7}$ Institute of \\ Pathology and Southwest Cancer Center, Southwest Hospital, Third Military Medical University (Army Medical University), Chongqing, China \\ Contributions: (I) Conception and design: P Gao, X Lin; (II) Administrative support: S Zhang; (III) Provision of study materials or patients: P Gao, W \\ Wu; (IV) Collection and assembly of data: All authors; (V) Data analysis and interpretation: P Gao, X Lin; (VI) Manuscript writing: All authors; (VII) \\ Final approval of manuscript: All authors. \\ "These authors contributed equally to this work. \\ Correspondence to: Xue Lin, MD; Shuyang Zhang, MD. Department of Cardiology, Peking Union Medical College Hospital, Chinese Academy of \\ Medical Sciences and Peking Union Medical College, Shuaifuyuan 1\#, East District, Beijing 100730, China. \\ Email: linxuepumch@qq.com; shuyangzhang103@nrdrs.org.
}

Background: Cardiovascular involvement manifesting as arrhythmias has been confirmed in patients with coronavirus disease 2019 (COVID-19), so we aimed to explore the association between primary tachyarrhythmia and death in critically ill patients with COVID-19 in this retrospective study.

Methods: A total of 79 critically ill patients with COVID-19 were included. Demographic characteristics, clinical data (past history, vital signs, therapeutic management, and outcomes), and results of laboratory findings and cardiac investigations were collected. All statistical analyses were performed using SPSS 23.0 software (IBM, Armonk, NY, USA).

Results: The median age was $65 \pm 12$ years, and 53 patients (67\%) were male. A total of $57(72 \%)$ patients died, and compared with survivors, these patients were older and had significantly higher Acute Physiology and Chronic Health Evaluation (APACHE) II score, Sequential Organ Failure Assessment (SOFA) score and fewer lymphocytes as well as higher heart rate $(\mathrm{P}<0.05)$. Autopsy findings did not suggest severe myocarditis. A total of 19 (24\%) patients had tachyarrhythmias, including $10(13 \%)$ with atrial fibrillation (AF) and $9(11 \%)$ with ventricular tachycardia or fibrillation. The incidence of tachyarrhythmias in non-survivor was much higher than in survivors $(\mathrm{P}=0.04)$. In a Cox regression model, older patients with ventricular tachyarrhythmias (VTAs) age were at a higher risk of death, with hazard ratio (HR) of 3.302 [95\% confidence interval (CI), 1.524-7.154, P=0.002] and 1.045 (95\% CI, 1.020-1.071, P=0.000), respectively. The use of beta-blockers [HR, 0.219 (95\% CI, 0.066-0.722); $\mathrm{P}=0.013$ ] was associated with a lower risk of death.

Conclusions: Critically ill patients with COVID-19 had a poor prognosis. VTA and older age were independent prognostic factors of death. Beta-blockers might be an effective therapy to improve survival.

Keywords: Arrhythmias; critically ill patients; mortality; coronavirus disease 2019 (COVID-19); beta-blockers

Submitted Apr 02, 2021. Accepted for publication May 21, 2021.

doi: 10.21037/atm-21-2282

View this article at: http://dx.doi.org/10.21037/atm-21-2282

(c) Annals of Translational Medicine. All rights reserved. 


\section{Introduction}

The outbreak of severe acute respiratory syndrome coronavirus 2 (SARS-CoV-2) infection [coronavirus disease 2019 (COVID-19) disease] has become a worldwide crisis (1-3). The virus has spread to more than 200 countries and territories, and two international conveyances. It is now known that the virus is highly contagious, and that most patients with mild symptoms are able to recover. However, once patients become critically ill, the mortality rate is $>50 \%$ (4-6). Although respiratory failure is the leading cause of death, organ failure, especially of the heart $(7,8)$, is also an independent cause of death. Recent studies have confirmed that myocardial injury is a high-risk factor for death (9). In addition, such patients also have a relatively high incidence of arrhythmia $(10,11)$. However, in critical ill patients, especially in those with well-supported vital signs, the sudden appearance of malignant arrhythmia may indicate a pathophysiological mechanism that has not been fully emphasized, so it has potential intervention value. Therefore, in this study, we focused on the relationship between tachyarrhythmia and mortality in critical ill patients.

We carried out a retrospective study to investigate the clinical characteristics of critically ill patients admitted to an intensive care unit (ICU) in Wuhan, China and assessed the role of tachyarrhythmias in all-cause death. We present the following article in accordance with the STROBE reporting checklist (available at http://dx.doi.org/10.21037/atm-21-2282).

\section{Methods}

\section{Study population}

A total of 79 consecutive critically ill patients with laboratory-confirmed COVID-19 and a positive CT scan of pneumonia were included in this retrospective cohort study. The patients were admitted between January 18th and March 12nd, 2020 to the ICU in Tongji Hospital (Sino-French New City campus), Huazhong University of Science and Technology, Wuhan. A multidisciplinary team from Peking Union Medical College (PUMC) Hospital, Beijing, consisting of doctors and nurses from the ICU and respiratory, infectious, cardiovascular, and nephrology departments, took care of these patients. The patients in this study were diagnosed according to the National Health Commission of the People's Republic of China: New Coronavirus Pneumonia Diagnosis and Treatment Plan (Trial Version 7) (12). Critically ill patients were defined as those with: (I) respiratory failure and requiring mechanical ventilation; (II) shock; and (III) other organ failure that required ICU care. The study was approved by the Ethical Committee of the PUMC Hospital (No. S-K 1297). Written informed consent was waived by the ethics committee of the PUMCH hospital for patients with emerging infectious diseases. All procedures performed in this study involving human participants were in accordance with the Declaration of Helsinki (as revised in 2013).

\section{Data collection}

The following data were collected from electronic medical records upon ICU admission: demographic characteristics (age and sex), clinical data (past history, vital signs, therapeutic management, and outcomes), and results of laboratory findings and cardiac investigations (cardiac biomarkers and electrocardiography monitoring). Atrial and ventricular arrhythmias were documented and analyzed separately. Atrial tachyarrhythmia was defined as atrial fibrillation (AF). Primary ventricular tachyarrhythmia (VTA) in this study was defined as a ventricular arrhythmia that suddenly appeared without obvious electrolyte imbalance under the condition of stable hemodynamics and respiratory function. Arrhythmia secondary to uncorrectable end-stage respiratory failure or infectious shock was excluded.

\section{Statistical analysis}

Continuous variables are presented as mean \pm standard deviation (SD) with a normal distribution or median (25-75th percentile) with a non-normal distribution. Two-sample t-test and Mann-Whitney $U$ test were used to assess the differences between survivors and non-survivors. The differences in categorical variables were tested using the $\chi^{2}$ test and Fisher's exact test (if the sample was $<5$ ). The relationship between two variables was analyzed using univariable correlated analysis. Cox regression was used for survival analysis. Survival time was defined as the period from admission until death or the end of follow-up. Two-sided $\mathrm{P}$ value $<0.05$ was considered significant. All statistical analyses were performed using SPSS 23.0 software (IBM, Armonk, NY, USA).

\section{Results}

\section{General characteristics of survivors and non-survivors}

A total of 79 patients with an average age of $65 \pm 12$ years 
were recruited, comprising 53 men $(66 \pm 12$ years) and 26 women ( $64 \pm 13$ years). At the end of follow-up, 22 patients survived and 57 had died. The overall mortality rate was $72 \%$ ( $81 \%$ for males and $54 \%$ for females, $\mathrm{P}=0.01)$. The course of the disease was $21[19,30]$ days from onset until death and $9[4,12]$ days from ICU admission to death. The causes of death were septic shock $(24,42 \%)$, respiratory failure $(20,35 \%)$, cardiac death $(10,18 \%)$, and stroke (3, $5 \%)$.

Compared with survivors, the non-survivors were older and predominantly male (Table 1). Hypertension was the most common coexisting condition, followed by diabetes, coronary heart disease, and stroke. Most of the patients had sinus tachycardia when admitted. The heart rate was significantly higher in the non-survival group than in the survival group.

A total of 77 patients $(97 \%)$ were diagnosed with acute respiratory distress syndrome (ARDS) according to the Berlin definition (13). Non-survivors had significantly higher Acute Physiology and Chronic Health Evaluation (APACHE) II and Sequential Organ Failure Assessment (SOFA) scores. In addition, the lymphocyte count was found to be significantly lower in non-survivors. Interestingly, the levels of high-sensitivity troponin I and $\mathrm{N}$-terminal proB-type natriuretic peptide (NT-proBNP) were slightly or moderately elevated, but showed no significant differences between survivors and non-survivors. Most of the patients, except $2(3 \%)$, had a history of prior myocardial infarction, but showed normal ejection fraction during bedside echocardiography.

With regard to treatment, $64(81 \%)$ patients were on an invasive mechanical ventilator. At the end of followup, only one survivor was still on a ventilator and receiving extracorporeal membrane oxygenation therapy. The use of mechanical ventilation, antiviral therapy, glucocorticoids, or tocilizumab (anti-human interleukin 6 receptor monoclonal antibody) was not significantly different between the two groups $(\mathrm{P}>0.05)$. However, more survivors were treated with beta-blockers, intravenous immunoglobulin, antibiotics, and anticoagulants than non-survivors (Table 2). Myocardial interstitial edema and small vasculitis were observed during autopsy, but there was neither significant inflammatory cell infiltration nor myocardial necrosis (Figure 1).

\section{Characteristics of patients with arrhythmia}

A total of 19 patients (24\%) had tachyarrhythmia, of whom $10(13 \%)$ had paroxysmal AF and $9(11 \%)$ had VTA. One of the 9 patients had a torsades de pointes pattern of ventricular tachycardia, and 8 had ventricular fibrillation; 2 (3\%) patients had both arrhythmias. Patients with $\mathrm{AF}$ were treated with medication (amiodarone) or

Table 1 Demographics and clinical characteristics of critically ill patients with COVID-19

\begin{tabular}{|c|c|c|c|c|}
\hline Variables & Overall group $(n=79)$ & Survivors ( $\mathrm{n}=22$ ) & Non-survivors $(n=57)$ & $P$ value \\
\hline Sex (male \%), n [\%] & $53[67]$ & $10[45]$ & $43[75]$ & 0.012 \\
\hline Age (years) & $66 \pm 12$ & $59 \pm 14$ & $68 \pm 10$ & 0.002 \\
\hline Survival (days) & $11[5,29]$ & $33[29,41]$ & $9[4,12]$ & 0.000 \\
\hline Smoking (\%), n [\%] & 30 [38] & $6[27]$ & 24 [42] & 0.303 \\
\hline \multicolumn{5}{|l|}{ Past history, n [\%] } \\
\hline CVD & $11[14]$ & $1[5]$ & $10[18]$ & 0.126 \\
\hline Hypertension, $\mathrm{n}$ [\%] & $40[51]$ & $10[45]$ & $30[53]$ & 0.622 \\
\hline Diabetes, n [\%] & 17 [22] & $6[28]$ & $11[19]$ & 0.543 \\
\hline COPD, n [\%] & $3[4]$ & $1[5]$ & $2[4]$ & 0.630 \\
\hline CKD, n [\%] & $4[5]$ & 1 [5] & $3[5]$ & 0.106 \\
\hline Stroke, n [\%] & $10[13]$ & 5 [23] & 5 [9] & 0.106 \\
\hline CHF, n [\%] & $2[3]$ & $0[0]$ & $2[4]$ & 0.518 \\
\hline Cancer, n [\%] & $2[3]$ & $0[0]$ & $2[4]$ & 0.518 \\
\hline
\end{tabular}

Table 1 (continued) 
Table 1 (continued)

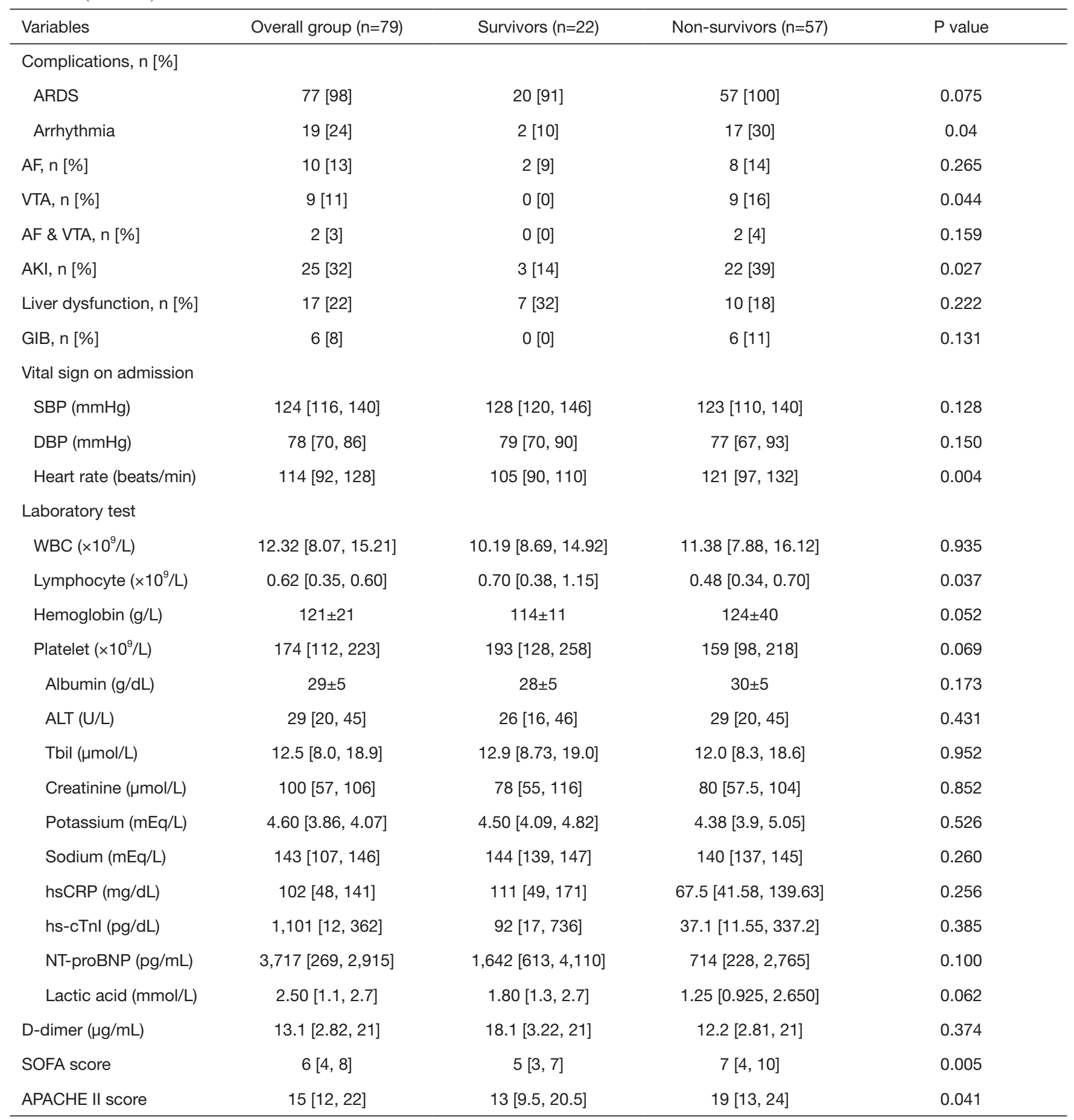

COVID-19, coronavirus disease 2019; CVD, cardiovascular disease; COPD, chronic obstructive pulmonary disease; CKD, chronic kidney disease; CHF, chronic heart failure; ARDS, acute respiratory distress syndrome; AF, atrial fibrillation; VTA, ventricular tachyarrhythmia; AKI, acute kidney injure; GIB, gastrointestinal bleeding; SBP, systolic blood pressure; DBP, diastolic blood pressure; WBC, white blood count; ALT, alanine aminotransferase; Tbil, total bilirubin; hsCRP, high-sensitivity C-reactive protein; hs-cTnl, hyper-sensitivity cardiac troponin I; NT-proBNP, N-terminal pro-brain natriuretic peptide; SOFA score, Sequential Organ Failure Assessment score; APACHE II score, Acute Physiology and Chronic Health Evaluation II score. 
Table 2 Treatment data of critically ill patients with COVID-19

\begin{tabular}{|c|c|c|c|c|}
\hline Variables & Overall group $(n=79)$ & Survivors $(\mathrm{n}=22)$ & Non-survivors $(n=57)$ & $P$ value \\
\hline Non-invasive mechanical ventilation, $\mathrm{n}$ [\%] & $24[30]$ & $7[32]$ & $17[30]$ & 1.000 \\
\hline Invasive mechanical ventilation, $\mathrm{n}$ [\%] & $66[84]$ & $17[77]$ & $49[86]$ & 0.499 \\
\hline Intubated on admission, $\mathrm{n}$ [\%] & $20[25]$ & $8[36]$ & $12[21]$ & 0.247 \\
\hline \multicolumn{5}{|l|}{ Medication, $\mathrm{n}[\%]$} \\
\hline Antiviral therapy & $56[71]$ & 18 [82] & $38[67]$ & 0.270 \\
\hline Glucocorticoid & $61[77]$ & 16 [73] & 45 [79] & 0.251 \\
\hline Intravenous immunoglobulin & 59 [75] & $21[95]$ & $38[67]$ & 0.000 \\
\hline Beta-blocker & 13 [16] & $10[45]$ & $3[5]$ & 0.000 \\
\hline Tocilizumab & 7 [9] & 2 [9] & $5[9]$ & 0.633 \\
\hline
\end{tabular}

COVID-19, coronavirus disease 2019.

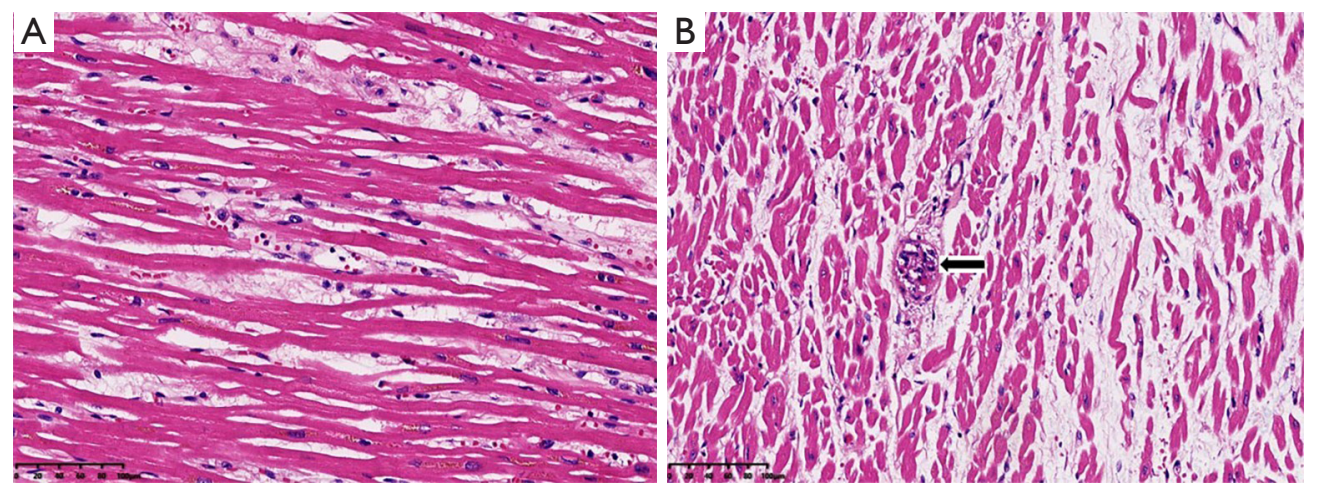

Figure 1 Autopsy results of the heart from a critically ill patient. (A) Left ventricular wall showing myocardial interstitial edema, and expanding spaces in the cardiac muscle. There is no significant inflammatory cell infiltration or myocardial necrosis (HE, $\times 200)$. (B) The main change in the left atrial wall is small-vessel vasculitis (arrow). The walls of small blood vessels thicken, become neutrophilic, and lymphocytic infiltration in onion skin like a vessel with lumen obstruction $(\mathrm{HE}, \times 200)$.

underwent electrical cardioversion immediately. None of the patients with VTA survived, even after electrical shock and resuscitation. The levels of hyper-sensitivity cardiac troponin I (hs-cTnI) and NT-proBNP when VTA occurred were not significantly different from those recorded upon admission $[653.2(76.4,1,035.5)$ vs. 361.6 (13.1, $701.25) \mathrm{pg} / \mathrm{mL} ; \mathrm{P}=0.581$ and $3,251(404,8,084)$ vs. 1,243 $(560,3,122) \mathrm{pg} / \mathrm{mL} ; \mathrm{P}=0.402$, respectively]. Transient sinus bradycardia was also observed in $4(5 \%)$ patients due to low body temperature, which was considered irrelevant to clinical outcomes.

Univariate correlation analysis indicated that tachyarrhythmia was related to male sex $(\mathrm{P}=0.005)$ and history of smoking $(\mathrm{P}=0.012)$. A correlation with higher heart rare on admission $(\mathrm{P}=0.055)$ was also found. The occurrence of tachyarrhythmia was not related to other clinical characteristics, comorbidities, laboratory indexes or therapies. 


\section{Arrhythmia and survival analysis}

Cox regression analysis was used to assess the relationship between arrhythmia with all-cause death. In the analysis model, patients with tachyarrhythmias (AF or VTA) had a higher risk of all-cause death $(\mathrm{P}=0.001)$. In particular, VTA significantly increased the risk of all-cause death $(\mathrm{P}=0.002)$, while AF did not $(\mathrm{P}=0.627)$. Age was another risk factor of death, whereas the use of beta-blockers was protective $(\mathrm{P}=0.013$; Table 3, Figure 2).

\section{Discussion}

The present study demonstrated a poor prognosis in critically ill patients admitted to the ICU and also analyzed

Table 3 HRs of risk factors in relation to death of critically ill patients with COVID-19

\begin{tabular}{lccc}
\hline Factors & $\mathrm{HR}$ & $95 \% \mathrm{Cl}$ & $\mathrm{P}$ value \\
\hline Age (years) & 1.045 & $1.020-1.071$ & 0.000 \\
Arrhythmia & & & 0.010 \\
AF & 1.210 & $0.561-2.609$ & 0.627 \\
VTA & 3.302 & $1.524-7.154$ & 0.002 \\
Beta-blockers & 0.219 & $0.066-0.722$ & 0.013 \\
\hline
\end{tabular}

HR, hazard ratio; COVID-19, coronavirus disease 2019; $\mathrm{Cl}$, confidence interval; AF, atrial fibrillation; VTA, ventricular tachyarrhythmia.

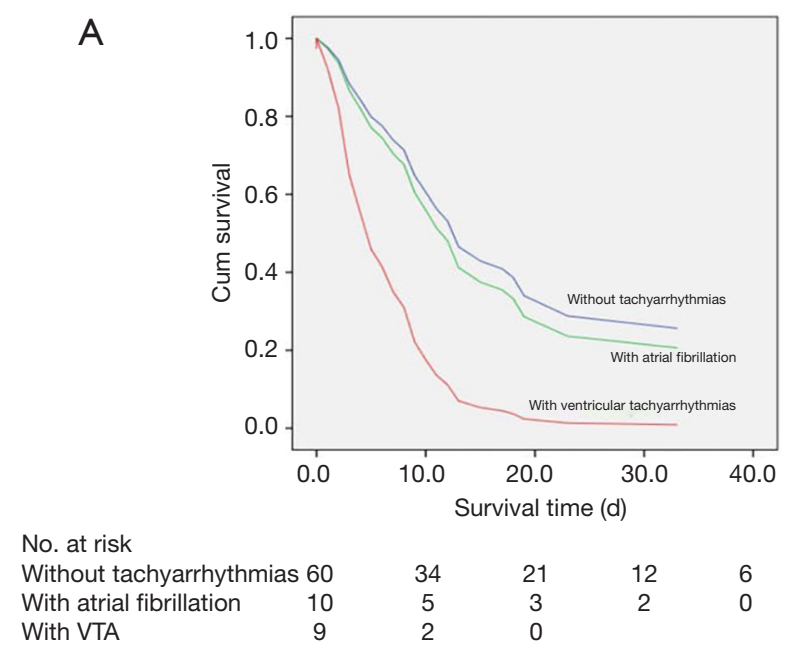

the risk factors of death. The global mortality rate is estimated to be $5.7 \%$ (14), but varies among countries. In China, $~ 5 \%$ of infected patients were admitted to ICUs and $2.3 \%$ were intubated. The most common significant complications were ARDS (15.6\%) and septic shock (6.4\%) in severe cases (2). In the present cohort of critically ill ICU patients, $84 \%$ needed invasive ventilation and had a high mortality rate of $72 \%$. These results were comparable to those of another study focusing on critically ill patients with $71 \%$ mechanical ventilation rate and $61.5 \%$ mortality rate (4). In addition to the generally recognized risk factors of older age, lymphopenia, high (SOFA) score, and cardiovascular commodities $(3,15,16)$, VTA was associated with poor outcomes and the use of beta-blockers had a protective role in these patients.

In a recent report from Wuhan, $16.7 \%$ of hospitalized and $44.4 \%$ of ICU patients with COVID-19 had arrhythmia (3). Also, there have been anecdotal reports of patients experiencing cardiac arrest with pulseless electrical activity or ventricular fibrillation during the recovery phase of their pulmonary illness. The present study showed that arrhythmia is common in critically ill patients with COVID-19 and is the third most common cause of death. VTA and older age were independent factors increasing allcause death. The use of beta-blockers might be a part of protective therapeutic management associated with lower mortality.

The reason for the rising incidence of arrhythmia is not apparent, but we speculate that it may be related to

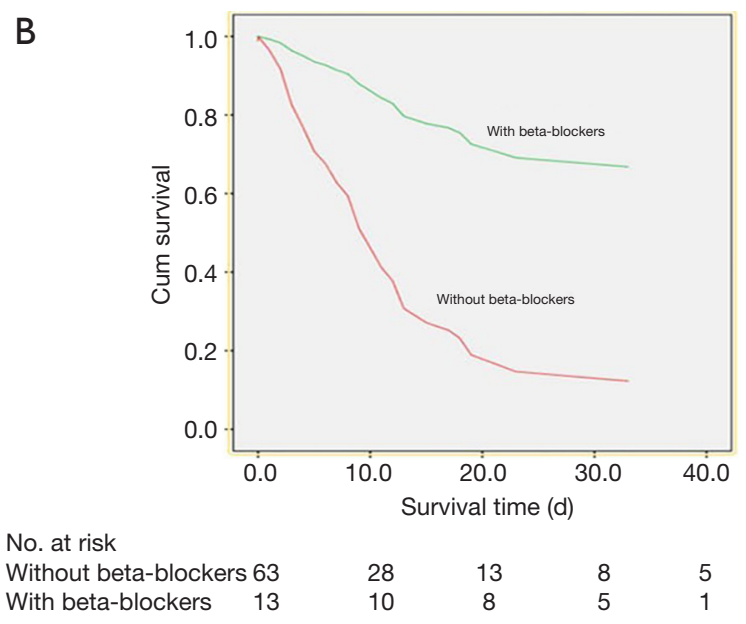

Figure 2 Cox proportional hazard regression analysis. (A) Risk in patients with VTA [HR, 3.302 (1.524-7.154); P=0.002], but AF does not show significance [HR, 1.210 (0.561-2.609); $\mathrm{P}=0.627]$. (B) Risk in patients with and without beta-blockers [HR, 0.219 (0.066-0.722); $\mathrm{P}=0.013]$. VTA, ventricular tachyarrhythmia; HR, hazard ratio; AF, atrial fibrillation. 
sympathetic overstimulation in the patients. The following three facts support this conclusion.

First, most of the patients had sinus tachycardia upon admission, especially those suffering from arrhythmia, which could be a consequence of poor clinical condition, including hypoxemia, fever, anemia, and inflammatory storm. An increased heart rate under stress is a sign of sympathetic nerve excitement, especially during critical illness. It is a physiological reaction allowing humans to survive during a crisis with the adrenergic-driven "fight-flight response". However, overstimulation of the sympathetic nervous system may have detrimental effects $(17,18)$, including tachycardia and tachyarrhythmia, myocardial ischemia, stunning, apoptosis, and necrosis. Sympathetic nerve excitement during respiratory failure leads to a compensatory increased heart rate that may be a precursor to malignant arrhythmia. Our conclusion was also supported by electric storm attacks observed in at least two patients with episodes of AF followed by VTA a few days later.

Second, VTA is difficult to explain by myocardial injury. Unlike most malignant arrhythmias originating from cardiac diseases, the arrhythmias in the current study were not related to the patients' pre-existing cardiac condition or the degree of myocardial injury. Recently, some studies have shown that myocardial injury occurring in $7-27.8 \%$ of hospitalized patients with COVID-19 significantly increased mortality $(3,9,15,16)$. A recent case report suggested fulminant myocarditis with no histological proof (19). There has been no evidence to elucidate causality between an elevated myocardial index and impending death. In the present study, the levels of troponin and NT-proBNP were slightly to moderately higher than the normal range, but showed no significant difference between survivors and nonsurvivors and had no apparent effect on prognosis in the survival analysis. Furthermore, almost all of the patients manifested absence of impaired left ventricular function on echocardiography. These findings indicated that most of the critically ill patients might have had different degrees of myocardial injury. However, myocardial injury may not be a factor affecting prognosis and heart function in critically ill patients. We speculate that even if myocardial injury predisposes to severe viral infection and deteriorating oxygen deficiency in critically ill patients, the myocardial injury is not the direct cause of death. Even though the indicators of myocardial damage were elevated, they did not reach the levels usually seen in myocardial infarction or fulminant myocarditis. Some of the autopsy results also confirmed this speculation. There were no apparent histological changes in cardiac tissues, suggesting that SARS-CoV-2 infection might not directly impair the heart $(20,21)$. Four patients in the current study had nearly normal histological findings without necrosis of cardiac muscle cells or inflammatory cell infiltration (Figure 1).

Third, the use of beta-blockers was significantly correlated with a reduction in the mortality rate. Betablockers are a classic medicine that reduces sympathetic excitability because they act upon $\beta$-adrenergic receptors, interfering with the ability of catecholamines or sympathomimetics. Some studies suggest that beta-blockers have a clinical value in patients with severe infections or ARDS, not only because they can reduce the excitability of sympathetic nerves and increase cardiac output, but because they also improve catalytic conditions (22). Appropriate timing and indications for the use of betablockers in COVID-19 remain important issues that need to be addressed via additional prospective studies. It is also worthwhile conducting large-scale clinical trials to confirm the role of beta-blockers. Based on our findings, we cautiously hypothesize that malignant arrhythmia in critical patients is related to increased sympathetic overstimulation and not to myocardial injury. Timely use of beta-receptor antagonists may help improve the prognosis. But, because of the limited numbers in this cohort and the difficulties associated with treating a highly contagious disease, more detailed data from other hospitals are needed for further assessment.

A relationship between $\mathrm{AF}$ and death was not observed in this study, possibly because immediate electrical or medical cardioversion was performed to achieve hemodynamic benefits. Another reason might lie in the small cohort size and the relatively mild effect on mortality. More extensive studies are needed to confirm whether $\mathrm{AF}$ affects prognosis.

\section{Conclusions}

Critically ill patients with COVID-19 admitted to the ICU showed poor prognosis. Arrhythmias were commonly seen among these patients, and VTA was an independent prognostic factor of death. Age was another risk factor. Beta-blockers were demonstrated to be a likely effective therapy to improve survival.

\section{Acknowledgments}

Funding: This work is sponsored by: the "13th Five-Year" 
National Science and Technology Major Project for New Drugs (No. 2019ZX09734001-002), the Chinese National Natural Science Foundation 81670349.

\section{Footnote}

Reporting Checklist: The authors have completed the STROBE reporting checklist. Available at http://dx.doi. org/10.21037/atm-21-2282

Data Sharing Statement: Available at http://dx.doi. org/10.21037/atm-21-2282

Conflicts of Interest: All authors have completed the ICMJE uniform disclosure form (available at http://dx.doi. org/10.21037/atm-21-2282). The authors have no conflicts of interest to declare.

Ethical Statement: The authors are accountable for all aspects of the work in ensuring that questions related to the accuracy or integrity of any part of the work are appropriately investigated and resolved. The present study was approved by the Ethical Committee of the PUMC Hospital (No. S-K 1297). Written informed consent was waived by the ethics committee of the PUMCH hospital for patients with emerging infectious diseases. All procedures performed in this study involving human participants were in accordance with the Declaration of Helsinki (as revised in 2013).

Open Access Statement: This is an Open Access article distributed in accordance with the Creative Commons Attribution-NonCommercial-NoDerivs 4.0 International License (CC BY-NC-ND 4.0), which permits the noncommercial replication and distribution of the article with the strict proviso that no changes or edits are made and the original work is properly cited (including links to both the formal publication through the relevant DOI and the license). See: https://creativecommons.org/licenses/by-nc-nd/4.0/.

\section{References}

1. Zhu N, Zhang D, Wang W, et al. A novel coronavirus from patients with pneumonia in China, 2019. N Engl J Med 2020;382:727-33.

2. Guan WJ, Ni ZY, Hu Y, et al. Clinical characteristics of coronavirus disease 2019 in China. N Engl J Med 2020;382:1708-20.
3. Wang D, Hu B, Hu C, et al. Clinical characteristics of 138 hospitalized patients with 2019 novel coronavirus-infected pneumonia in Wuhan, China. JAMA 2020;323:1061-9. Erratum in: JAMA 2021;325:1113.

4. Yang X, Yu Y, Xu J, et al. Clinical course and outcomes of critically ill patients with SARS-CoV-2 pneumonia in Wuhan, China: a single-centered, retrospective, observational study. Lancet Respir Med 2020;8:475-81. Erratum in: Lancet Respir Med 2020;8:e26.

5. Arentz M, Yim E, Klaff L, et al. Characteristics and outcomes of 21 critically ill patients with COVID-19 in Washington State. JAMA 2020;323:1612-4.

6. Murthy S, Gomersall CD, Fowler RA. Care for critically ill patients with COVID-19. JAMA 2020;323:1499-500.

7. He XW, Lai JS, Cheng J, et al. Impact of complicated myocardial injury on the clinical outcome of severe or critically ill COVID-19 patients. Zhonghua Xin Xue Guan Bing Za Zhi 2020;48:456-60.

8. Vagnozzi RJ, Maillet M, Sargent MA, et al. An acute immune response underlies the benefit of cardiac stem cell therapy. Nature 2020;577:405-9.

9. Shi S, Qin M, Shen B, et al. Association of cardiac injury with mortality in hospitalized patients with COVID-19 in Wuhan, China. JAMA Cardiol 2020;5:802-10.

10. Madjid M, Safavi-Naeini P, Solomon SD, et al. Potential effects of coronaviruses on the cardiovascular system: a review. JAMA Cardiol 2020;5:831-40.

11. Guo T, Fan Y, Chen M, et al. Cardiovascular implications of fatal outcomes of patients with coronavirus disease 2019 (COVID-19). JAMA Cardiol 2020;5:811-8. Erratum in: JAMA Cardiol 2020;5:848.

12. National Health Commission of People's Republic of China. Diagnosis and Treatment Protocol for Novel Coronavirus Pneumonia (Trial Version 7). Published March 3, 2020. Available online: https://www.chinadaily. com.cn/pdf/2020/1.Clinical.Protocols.for.the.Diagnosis. and.Treatment.of.COVID-19.V7.pdf

13. Ranieri VM, Rubenfeld GD, Thompson BT, et al. Acute respiratory distress syndrome: the Berlin Definition. JAMA 2012;307:2526-33.

14. Baud D, Qi X, Nielsen-Saines K, et al. Real estimates of mortality following COVID-19 infection. Lancet Infect Dis 2020;20:773.

15. Zhou F, Yu T, Du R, et al. Clinical course and risk factors for mortality of adult inpatients with COVID-19 in Wuhan, China: a retrospective cohort study. Lancet 2020;395:1054-62. Erratum in: Lancet 2020;395:1038.

16. Huang C, Wang Y, Li X, et al. Clinical features of patients 
infected with 2019 novel coronavirus in Wuhan, China. Lancet 2020;395:497-506. Erratum in: Lancet 2020.

17. Bucsek MJ, Giridharan T, MacDonald CR, et al. An overview of the role of sympathetic regulation of immune responses in infectious disease and autoimmunity. Int $\mathrm{J}$ Hyperthermia 2018;34:135-43.

18. Andreis DT, Singer M. Catecholamines for inflammatory shock: a Jekyll-and-Hyde conundrum. Intensive Care Med 2016;42:1387-97.

19. Hu H, Ma F, Wei X, et al. Coronavirus fulminant myocarditis saved with glucocorticoid and human immunoglobulin. Eur Heart J 2021;42:191.

Cite this article as: Gao P, Wu W, Tian R, Yan X, Qian H, Guo F, Li T, Liu Z, Wang J, Zhou X, Qin Y, Zhao D, Bian X, Lin $\mathrm{X}$, Zhang S. Association between tachyarrhythmia and mortality in a cohort of critically ill patients with coronavirus disease 2019 (COVID-19). Ann Transl Med 2021;9(10):883. doi: 10.21037/atm-21-2282
20. Liu Q, Wang RS, Qu GQ, et al. Gross examination report of a COVID-19 death autopsy. Fa Yi Xue Za Zhi 2020;36:21-3.

21. Wang HJ, Du SH, Yue X, et al. Review and prospect of pathological features of corona virus disease. Fa Yi Xue Za Zhi 2020;36:16-20.

22. Coppola S, Froio S, Chiumello D. $\beta$-blockers in critically ill patients: from physiology to clinical evidence. Crit Care 2015;19:119.

(English Language Editor: K. Brown) 